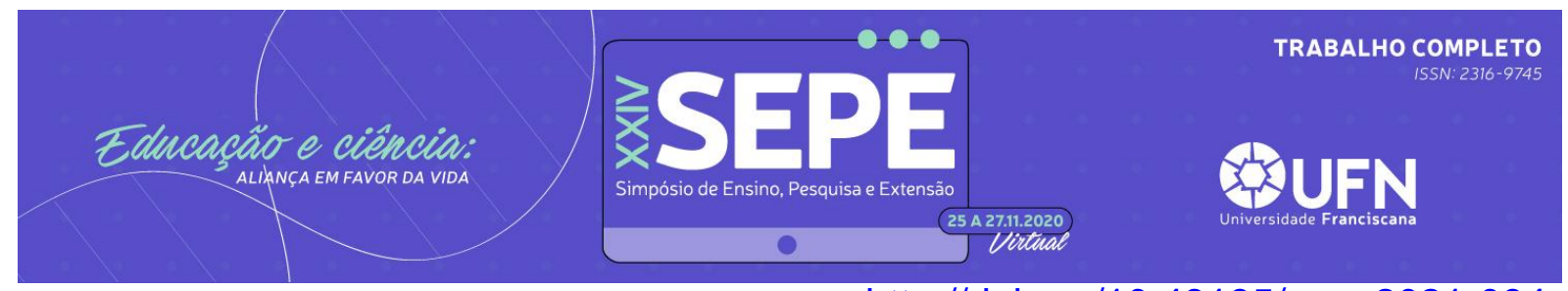

http://doi.org/10.48195/sepe2021-094

\title{
FRENOTOMIA E SEU IMPACTO NA AMAMENTAÇÃO DOS BEBÊS
}

\section{Ana Carolina Zen Rossato', Letícia Mendes', Cristiane Medianeira Savian², Cândida Bonadiman ${ }^{3}$, Maurício Barbieri Mezomo4, Letícia Westphalen Bento ${ }^{4}$, Simone Pippi Antoniazzi ${ }^{4}$, Bianca Zimmermann Santos ${ }^{5}$}

\footnotetext{
${ }^{1}$ Acadêmica do Curso de Odontologia - Universidade Franciscana - UFN

2 Mestre em Saúde Materno Infantil - Universidade Franciscana - UFN

${ }^{3}$ Mestranda em Saúde Materno Infantil - Universidade Franciscana - UFN

${ }^{4}$ Professor(a) do Curso de Odontologia - Universidade Franciscana - UFN

${ }^{5}$ Orientadora. Professora do Curso de Odontologia e do Mestrado em Saúde Materno Infantil Universidade Franciscana - UFN
}

\section{RESUMO}

O objetivo desta revisão de literatura é descrever sobre a frenotomia e suas implicações na amamentação dos bebês. As buscas foram realizadas nas bases de dados MEDLINE/PubMed com os descritores MeSH "infant", "lingual frenum", e "ankyloglossia" e para buscas na base de dados Scielo utilizou-se os seguintes descritores DeCS: "lactente", "freio lingual" e "anquiloglossia", combinados pelo operador booleano AND. Não foi realizada a delimitação temporal. Foram incluídos somente artigos que contemplassem a temática proposta. As buscas foram realizadas no mês de julho de 2021. A anquiloglossia é caracterizada pela presença do freio lingual curto e está associada com as dificuldades de aleitamento materno. Para estabelecer um diagnóstico mais preciso de anquiloglossia é necessária uma equipe multidisciplinar e da realização de protocolos do teste da linguinha. A frenotomia consiste em um procedimento cirúrgico indicado para ampliar a inserção do freio lingual em bebês, podendo ser realizado em nível ambulatorial por cirurgiões dentistas, médicos pediatras e otorrinolaringologistas. $O$ aleitamento materno é a mais completa e natural forma de criação de vínculo, afeto, proteção e nutrição para o bebê, sendo o mais sensível, econômico e eficaz método para redução da morbimortalidade infantil. Permite ainda um grande impacto na promoção da saúde integral do binômio mamãe/bebê e de toda a família. Para isso é importante e necessário o diagnóstico precoce e tratamento oportuno dos fatores que possam interromper o aleitamento materno.

Palavras-chave: lactente, anquiloglossia, amamentação.

Eixo Temático: Saúde Materno Infantil (SMI).

\section{INTRODUÇÃO}




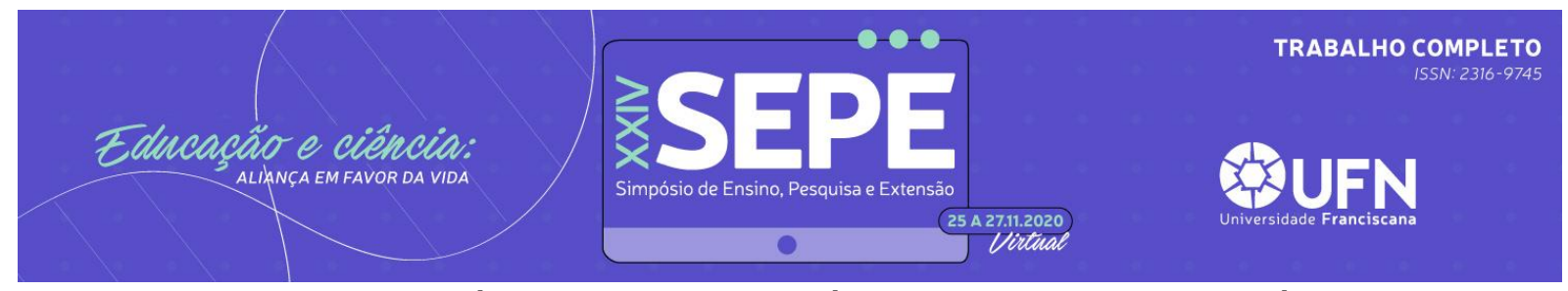

A anquiloglossia ou língua presa, como é comumente chamada, é uma anomalia de desenvolvimento da língua caracterizada por um freio lingual curto (freio lingual é uma pequena membrana que fica abaixo da língua e impede que ela se movimente adequadamente), que resulta na limitação dos movimentos da mesma (WALSH; TUNKEL, 2017). Apresenta etiologia indefinida e não mostra predileção por gênero (SILVA et al., 2019). A anquiloglossia tem associação direta com a amamentação, pois impede que o recém-nascido execute os movimentos corretos da ordenha e sucção, podendo gerar dor, infecção mamilar, interrupção da amamentação, diminuição da produção de leite materno, perda de peso do bebê e até mesmo dificuldade da higiene bucal do recém-nascido (FUJINAGA et al., 2017).

Sendo assim, é necessário que sejam realizadas ações integrativas para o correto diagnóstico e decisão cirúrgica precoce visando a qualidade de vida no binômio mamãe-bebê (FUJINAGA et al., 2017). Para estabelecer um diagnóstico mais preciso de anquiloglossia é necessária uma equipe multidisciplinar com médicos, odontopediatras, enfermeiros e fonoaudiólogos. Para o correto diagnóstico recomenda-se a realização do teste da linguinha (MARTINELLI; MARCHESAN; BERRETIN-FELIX, 2012) ou teste de Bristol Tongue Assessment Tool (BTAT) (INGRAM et al., 2015), que também pode ser utilizado para a identificação da anquiloglossia. Recomenda-se que a avaliação do frênulo da língua seja inicialmente realizada na maternidade. A avaliação precoce é ideal para que os bebês sejam diagnosticados e tratados corretamente (MARTINELLI; MARCHESAN; BERRETINFELIX 2012).

O tratamento para a anquiloglossia é a frenotomia (procedimento cirúrgico realizado para reposicionar o frênulo língual, sem remoção de tecido, apenas a reinserção do mesmo) ou a frenectomia (procedimento cirúrgico realizado para remoção do frênulo lingual, com remoção de tecido) (VIEIRA; MACHADO, 2018). A frenotomia pode ser realizada, preferencialmente, nos primeiros dias ou meses de vida por dentistas, médicos pediatras ou otorrinolaringologistas habilitados. É um procedimento rápido, seguro e eficaz que pode ter influência significativa na amamentação, possibilitando o correto encaixe da língua e consequente alívio de desconfortos e traumas mamilares (LIMA; DUTRA, 2021). 


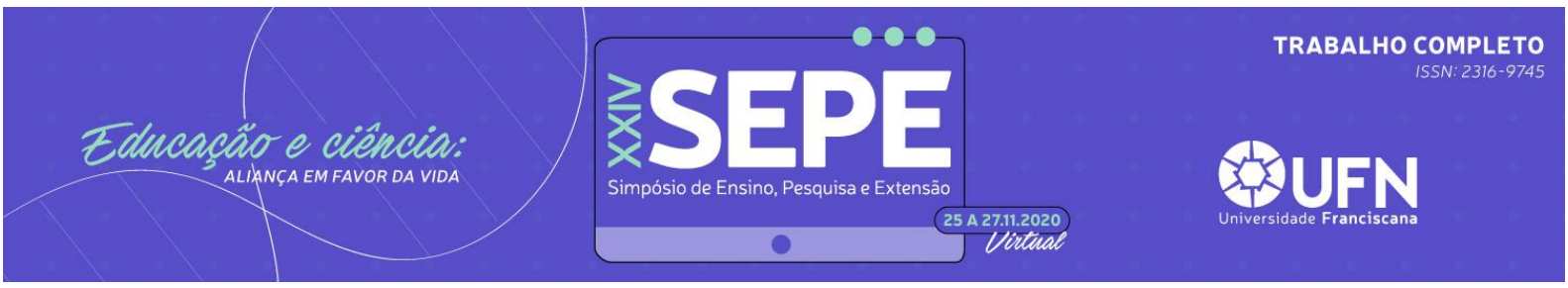

Considerando a importância do tema para a saúde materno infantil o objetivo desta revisão de literatura é descrever sobre a frenotomia e suas implicações na amamentação dos bebês.

\section{METODOLOGIA}

O estudo corresponde a uma revisão narrativa de literatura e para o desenvolvimento desta, foi realizada uma busca de artigos referentes ao tema estudado, nas bases de dados MEDLINE/PubMed e Scielo. A pesquisa foi realizada no mês de julho de 2021. Foram utilizados os seguintes descritores do MeSH: "infant", "lingual frenum", "ankyloglossia" e para buscas na base de dados Scielo utilizou-se os seguintes descritores DeCS: "lactente", "freio lingual" e "anquiloglossia", combinados pelo operador booleano AND. Não foi realizada a delimitação temporal visando obter todos os trabalhos já desenvolvidos sobre o assunto na base de dados citada.

Foi realizada a leitura dos títulos e resumos de todos os artigos encontrados, para adequada inclusão dos trabalhos relacionados ao tema. Como critério de inclusão foi considerado: artigos que contemplassem a temática proposta, publicados em português, inglês e espanhol. O critério de exclusão estabelecido foi artigos que não estivessem com o texto completo disponível. As referências dos artigos incluídos também foram revisadas para encontrar possíveis artigos que pudessem ser incluídos.

\section{RESULTADOS E DISCUSSÃO}

O frênulo lingual consiste em uma pequena prega de membrana mucosa que faz a conexão do assoalho oral à porção inferior da língua (linha média). A anquiloglossia é uma anomalia de desenvolvimento que envolve o frênulo lingual, caracterizada pelo seu encurtamento e/ou aumento de espessura, assim como inserção anteriorizada, próximo à ponta da língua, limitando os movimentos normais da língua (GOMES et al., 2015). A anquiloglossia é hereditária, a maioria dos estudos, relatos de casos, está relacionada a parentes próximos, como a mãe e o pai (LIMA; DUTRA, 2021). 


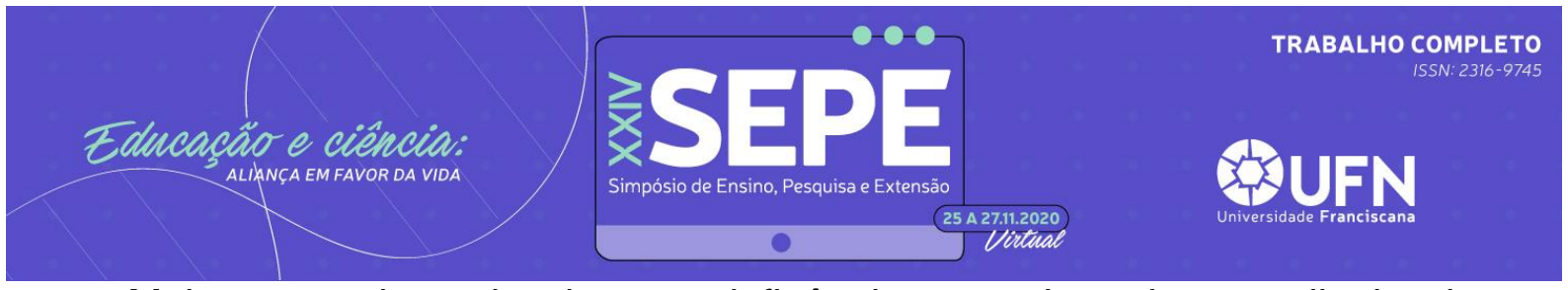

Muitos estudos abordam a influência negativa da anquiloglossia na amamentação dos recém-nascidos (SILVA et al., 2019; FRAGA et al., 2021). A Organização Mundial da Saúde (OMS) recomenda o aleitamento materno exclusivo nos seis primeiros meses de vida do bebê, pois esta auxilia no crescimento e desenvolvimento da criança, diminuindo os riscos de doenças típicas da infância, como diabetes, obesidade, anemia, entre outras (BRASIL, 2016).

A correta protusão lingual e o selamento da aréola mamária são fatores essenciais para uma correta amamentação. A limitação nos movimentos da língua em bebês com anquiloglossia pode prejudicar as funções de sucção e deglutição, que estão diretamente relacionadas à amamentação, além de interferir no correto posicionamento e encaixe da boca do bebê na mama, podendo causar fissuras mamilares, sensação de picada e dores (LIMA; DUTRA, 2021).

Alguns dos fatores relacionados ao desmame precoce, considerando, além dos fatores relacionados à mãe, como o baixo nível educacional e socioeconômico, os que são relacionados ao recém-nascido, como a pega incorreta do peito e a presença de anquiloglossia (FRAGA et al., 2021). Assim, enfatiza-se a importância do diagnóstico precoce de anquiloglossia como forma de evitar o desmame precoce, especialmente em populações de maior vulnerabilidade econômica e social, para manter o aleitamento artificial (LIMA; DUTRA, 2021).

O diagnóstico da anquiloglossia em recém-nascidos pode sofrer variação confome instrumento de avaliação utilizado (FRAGA et al., 2021). Diante disso, no ano de 2014, foi aprovada a Lei Federal no 13.002, que preconiza a obrigatoriedade da aplicação do Protocolo de Avaliação do Frênulo da Língua em Bebês nos recémnascidos de todas as maternidades do Brasil. Esse protocolo pode ser aplicado por profissionais de saúde habilitados, dentre eles podem estar presentes o dentista, 0 fonoaudiólogo, o enfermeiro e o médico (BRASIL, 2014). Quando, mesmo após a aplicação do Protocolo de Avaliação do Frênulo da Língua para Bebês, ainda na maternidade restaram dúvidas quanto a presença de anquiloglossia, o bebê deve passar por uma reavaliação após 30 dias de vida, de forma a considerar as dificuldades apresentadas na amamentação para a interpretação dos resultados e a indicação do tratamento (FRAGA et al., 2021). 
Após a confirmação do diagnóstico da anquiloglossia, a frenotomia ou a frenotomia são indicadas. A frenotomia é uma técnica cirúrgica pouco invasiva, com expectativa de dor pós-operatória leve, indicando-se assim a amamentação imediata o pós-operatório. É eficiente para melhora da postura, mobilidade da língua e suas funções (BISTAFFA et al., 2017). Durante a cirurgia lingual, reposiciona-se o freio permitindo a liberação dos movimentos da língua. O método cirúrgico mais utilizado é com o uso de bisturi ou tesoura reta, porém atualmente estudos mostram uma nova técnica utilizando lasers de alta potência de CO2 (CASTRO-RODRÍQUEZ et al., 2020). A percepção de dor pós-operatória é menor na técnica com laser de $\mathrm{CO}_{2}$ do que nas técnicas com bisturi ou tesoura. $O$ tempo cirúrgico, também, é significativamente menor (SILVA et al., 2018).

No estudo realizado por Siqueira e colaboradores (2020), os resultados alcançados após a técnica da frenotomia lingual em recém-nascidos, demonstrou resultados positivos na melhora da amamentação e a consequente ampliação do vínculo maternoinfantil. Além disso, houve melhora da "pega" do recém-nascido no mamilo e consequente redução do trauma e da dor mamilar, referidos pelas mães. Sendo assim, o diagnóstico precoce e a intervenção para a anquiloglossia são importantes para o sucesso do aleitamento materno, e todos os benefícios que este traz para o binômio mamãe/bebê.

\section{CONCLUSÃO}

O aleitamento materno é a mais completa e natural forma de criação de vínculo, afeto, proteção e nutrição para o bebê, sendo o mais sensível, econômico e eficaz método para redução da morbimortalidade infantil. Permite ainda um grande impacto na promoção da saúde integral do binômio mamãe/bebê e de toda a família. Para isso é importante e necessário o diagnóstico precoce e tratamento oportuno dos fatores que possam interromper o aleitamento materno, como o freio lingual curto. Nesse sentido o procedimento de frenotomia mostra-se seguro e efetivo. 


\section{REFERÊNCIAS}

BISTAFFA, A. G. I.; GIFFONI, T. C. R.; FRANZIN, L. C. S. Frenotomia lingual em bebê. Revista Uningá Review, v. 29, n. 2, 2017.

BRASIL. Lei 13.002, de 20 de junho de 2014. Obriga a realização do Protocolo de Avaliação do Frênulo da Língua em Bebês. 2014.

BRASIL. Ministério da Saúde. Saúde da Criança. Aleitamento Materno e Alimentação Complementar. Cadernos de Atenção Básica, ํำ 23. 2ª edição. 2016.

CASTRO-RODRÍGUEZ, Y.; BRAVO-CASTAGNOLA, F.; GRADOS-POMARINO, S. Resultados clínicos del tratamiento de frenillos labiales; frenectomía y frenotomía. International Journal of Interdisciplinary Dentistry, v. 13, n. 1, p. 4043, 2020.

FRAGA, M. R. B. A. et al. Diagnosis of ankyloglossia in newborns: is there any difference related to the screening method. CoDAS, n. 33, n. 1:e20190209, p. 1-7, 2021.

FUJINAGA, C. I. et al. Frênulo lingual e aleitamento materno: estudo descritivo. Audiology-Communication Research, v. 22, n. e1762, p. 1-5, 2017.

GOMES, E.; ARAÚJO, F. B.; RODRIGUES, J. A. Freio lingual: abordagem clínica interdisciplinar da Fonoaudiologia e Odontopediatria. Revista da Associação Paulista de Cirurgiões Dentistas, v. 69, n. 1, p. 20-24, 2015.

INGRAM, J. et al. The development of a tongue assessment tool to assist with tonguetie identification. Archives of Disease in Childhood, v. 100, n. 4, p. 344-348, 2015. 


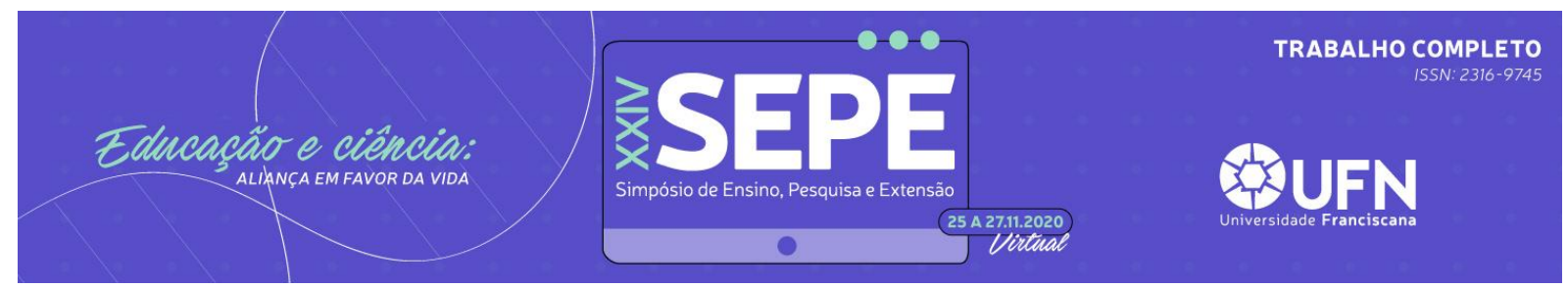

LIMA, A. L. X.; DUTRA, M. R. P. Influence of frenotomy on breastfeeding in newborns with ankyloglossia. CoDAS, v. 33, n. 01, p. 1-5, 2021.

MARTINELLI, R. L. C.; MARCHESAN, I. Q.; BERRETIN-FELIX, G. Lingual frenulum protocol with scores for infants. The International Journal of Orofacial Myology, v. 38, n. 1, p. 104-112, 2012.

SILVA, H. L.; SILVA, J. J.; ALMEIDA, L. F. Frenectomia: revisão de conceitos e técnicas cirúrgicas. SALUSVITA, v. 37, n. 1, p. 139-150, 2018.

SILVA, G. F.; CUMINI, P. P.; FARIA, M. D. Anquiloglossia em bebês: revisão de literatura. ANAIS DE ODONTOLOGIA DO UNIFUNEC, v. 6, n. 6, 2019.

SIQUEIRA, B. et al. Oral health of neonates: mothers' perception of lingual frenotomy performed in a university hospital. RGO-Revista Gaúcha de Odontologia, v. 68, n. e20200023, p. 1-7, 2020.

VIEIRA, K. A.; MACHADO, F. G. Frenectomia Em Odontopediatria: Relato De Caso. Revista da ACBO, v. 7, n. 2, p. 145-152, 2018.

WALSH, J.; TUNKEL, D. Diagnosis and Treatment of Ankyloglossia in Newborns and Infants: A Review. JAMA Otolaryngology-Head \& Neck Surgery, v. 13, p. 1-8, 2017. 